\title{
Flexural motions under moving concentrated masses of elastically supported rectangular plates resting on va- riable winkler elastic foundation
}

\section{Abstract}

The flexural motions of elastically supported rectangular plates carrying moving masses and resting on variable Winkler elastic foundations is investigated in this work In order to solve the fourth order partial differential equation governing the problem, a technique based on separation of variables is used to reduce the governing fourth order partial differential equations with variable and singular coefficients to a sequence of second order ordinary differential equations. These equations are then solved using a modification of the Struble's technique and method of integral transformations. Numerical results are then presented in plotted curves. The results show that response amplitudes of the plate decrease as the value of the rotatory inertia correction factor $R_{0}$ increases and for fixed value of $R_{0}$, the displacements of the elastically supported rectangular plates resting on variable elastic foundations decrease as the foundation modulus $F_{o}$ increases. Also, for fixed $R_{0}$ and $F_{o}$, the transverse deflections of the rectangular plates under the actions of moving masses are higher than those when only the force effects of the moving load are considered. Therefore, the moving force solution is not a safe approximation to the moving mass problem. Hence, safety is not guaranteed for a design based on the moving force solution. Furthermore, the results show that the critical speed for the moving mass problem is reached prior to that of the moving force for the elastically supported rectangular plates on Winkler elastic foundation with stiffness variation.

\section{Keywords}

Winkler Foundation, Foundation Modulus, Rotatory Inertia, Resonance, Critical Speed, Moving Force, Moving Mass.

\section{T. O. Awodola*}

Department of Mathematical Sciences Federal University of Technology, Akure, Nigeria

*Author email: oluthomas71@yahoo.com 


\section{INTRODUCTION}

The analyses of elastic structures, such as beams and plates, acted upon by moving loads and resting on a foundation constitute an important part of Engineering and applied Mathematics literatures. In general, such analyses are mathematically complex due to the difficulty in modeling the mechanical response of the subgrade which is governed by many factors.

When the vehicle-track interaction is completely neglected, we have the so called 'moving force' problem which has been shown by several researchers that it is a crude approximation to the 'moving mass' problem where the vehicle-track interaction is considered, Muscolino and Palmeri (2007). Several researchers have considered the vehicle-track interaction in their analyses. These researchers include Stanisic et al (1974), Milornir et al (1969), Clastornic et al (1986), Sadiku and Leipholz (1981) and Gbadeyan and Oni (1995). Douglas et al (2002) solved the problem of plate strip of varying thickness and the center of shear. In their work, they considered a free-vibrating strip with classical boundary conditions, precisely, they assumed the plate strip clamped at one end and free at the other end. Pesterev et al (2001) came up with a series expansion method for calculating bending moment and shear force in the problem of vibration of a damped beam subject to an arbitrary number of moving loads. This kind of solution, though could be accurate, cannot account for vital information such as the phenomenon of resonance in the dynamical system.

Recently, several other researchers have made tremendous efforts in the study of dynamics of structures under moving loads, these include Oni (2004), Oni and Omolofe (2005), Oni and Awodola (2003), Omer and Aitung (2006), Adams (1995), Savin (2001), Jia-Jang (2006). In all of these, considerations have been limited to cases of one-dimensional (beam) problems. Where twodimensional (plate) problems have been considered, the foundation moduli are taken to be constants. No considerations have been given to the class of dynamical problems in which the foundation is the type with stiffness variation. In an attempt to solve such two-dimensional problem, all the methods used in the above works break down due to the variation of the foundation model.

Generally, the dynamical problems of structures under moving load and resting on a foundation is complex, the complexity increases if the foundation stiffness varies along the structure. Aside the problem of singularity brought in by the inclusion of the inertia effects of the moving load, the coefficients of the governing fourth order partial differential equation are no longer constant but variable. Earlier researchers into beam member on variable elastic foundation include Franklin and Scott (1979) who presented a closed-form solution to a linear variation of the foundation modulus using contour-integrals. In a recent development, Oni and Awodola (2005) investigated the dynamic response to moving concentrated masses of uniform Rayleigh beams resting on variable Winkler elastic foundation.

However, in all these, the problem of determining the dynamic response of structures under the action of moving concentrated masses has been almost exclusively reserved for elastic structures having the normal ideal boundary conditions. Such ideal boundary conditions include among others, Clamped edge, Free edge, Simply supported edge and Sliding edge boundary conditions. For practical applications in many cases, it is more realistic to consider non-classical boundary conditions because the ideal boundary conditions can seldom be realized. A common 
example is the elastically supported end conditions. As a problem of this kind, Wilson (1974) studied the response of a cantilever plate strip restrained elastically against rotation and subjected to a moving normal line load.

More recently, Oni and Awodola (2010) considered the dynamic response under a moving load of an elastically supported non-prismatic Bernoulli-Euler beam on variable elastic foundation. The technique was based on the generalized Galerkin's method and integral transformations.

In all these previous investigations, extension of the theory to cover two-dimensional (plate) problem has not been effected, when the plate is on variable foundation. Therefore, this study concerns the response to moving concentrated masses of elastically supported rectangular plate resting on Winkler elastic foundation with stiffness variation.

\section{GOVERNING EQUATION}

Consider a rectangular plate carrying an arbitrary number (say $\mathrm{N}$ ) of concentrated masses $\mathrm{M}_{\mathrm{i}}$ moving with constant velocities $\mathrm{c}_{\mathrm{i}}, \mathrm{i}=1,2,3, \ldots, \mathrm{N}$ along a straight line parallel to the $\mathrm{x}$ - axis ( no difficulty arises by assuming that masses travel in an arbitrary path ) issuing from point $\mathrm{y}=$ $\mathrm{s}$ on the $\mathrm{y}-$ axis. The equation governing the dynamic transverse displacement $\mathrm{W}(\mathrm{x}, \mathrm{y}, \mathrm{t})$ of an elastically supported rectangular plate when it is resting on a variable Winkler foundation and traversed by several moving concentrated masses is the fourth order partial differential equation given by; Oni and Awodola (2011),

$$
\begin{aligned}
& D \nabla^{4} W(x, y, t)+\mu \frac{\partial^{2} W(x, y, t)}{\partial t^{2}}=\mu R_{0}\left[\frac{\partial^{4}}{\partial t^{2} \partial x^{2}}+\frac{\partial^{4}}{\partial t^{2} \partial y^{2}}\right] W(x, y, t) \\
& -F_{0}\left[4 x-3 x^{2}+x^{3}\right] W(x, y, t)+\sum_{i=1}^{N}\left[M_{i} g \delta\left(x-c_{i} t\right) \delta(y-s)\right. \\
& \left.-M_{i}\left(\frac{\partial^{2}}{\partial t^{2}}+2 c_{i} \frac{\partial^{2}}{\partial t \partial x}+c_{i}^{2} \frac{\partial^{2}}{\partial x^{2}}\right) W(x, y, t) \delta\left(x-c_{i} t\right) \delta(y-s)\right]
\end{aligned}
$$

where

$$
D=\frac{E h^{2}}{12(1-v)}
$$

is the bending rigidity of the plate, $\nabla^{2}$ is the two-dimensional Laplacian operator, $\mathrm{h}$ is the plate's thickness, E is the Young's Modulus, $v$ is the Poisson's ratio $(v<1), \mu$ is the mass per unit area of the plate, $R_{0}$ is the Rotatory inertia correction factor, $\mathrm{F}_{0}$ is the foundation's stiffness, $\mathrm{g}$ is the acceleration due to gravity, $\mathrm{x}$ and $\mathrm{y}$ are respectively the spatial coordinates in $\mathrm{x}$ and $\mathrm{y}$ directions and $\mathrm{t}$ is the time coordinate. $\delta($.$) is the Dirac - Delta function.$

The initial conditions, without any loss of generality, is taken as 


$$
W(x, y, t)=0=\frac{\partial W(x, y, t)}{\partial t}
$$

In this paper, in the first instance, we consider rectangular plate resting on a variable Winkler elastic foundation elastically supported at edges $\mathrm{y}=0, \mathrm{y}=\mathrm{L}_{\mathrm{Y}}$ with simple support at edges $\mathrm{x}=$ $0, \mathrm{x}=\mathrm{L}_{\mathrm{X}}$, the boundary conditions can be written as; Oni and Awodola (2010)

$$
\begin{aligned}
W(0, y, t) & =0, & W\left(L_{X}, y, t\right)=0 \\
\frac{\partial^{2} W(x, 0, t)}{\partial y^{2}}-k_{1} \frac{\partial W(x, 0, t)}{\partial y} & =0, & \frac{\partial^{2} W\left(x, L_{Y}, t\right)}{\partial y^{2}}-k_{1} \frac{\partial W\left(x, L_{Y}, t\right)}{\partial y}=0 \\
\frac{\partial^{2} W(0, y, t)}{\partial x^{2}} & =0, & \frac{\partial^{2} W\left(L_{X}, y, t\right)}{\partial x^{2}}=0 \\
\frac{\partial^{3} W(x, 0, t)}{\partial y^{3}}+k_{2} W(x, 0, t) & =0, & \frac{\partial^{3} W\left(x, L_{Y}, t\right)}{\partial y^{3}}+k_{2} W\left(x, L_{Y}, t\right)=0
\end{aligned}
$$

and for normal modes

$$
\begin{aligned}
\Psi_{n i}(0)=0, & \Psi_{n i}\left(L_{X}\right)=0 \\
\frac{\partial^{2} \Psi_{n j}(0)}{\partial y^{2}}-k_{1} \frac{\partial \Psi_{n j}(0)}{\partial y}=0, & \frac{\partial^{2} \Psi_{n j}\left(L_{Y}\right)}{\partial y^{2}}-k_{1} \frac{\partial \Psi_{n j}\left(L_{Y}\right)}{\partial y}=0 \\
\frac{\partial^{2} \Psi_{n i}(0)}{\partial x^{2}}=0, & \frac{\partial^{2} \Psi_{n i}\left(L_{X}\right)}{\partial x^{2}}=0 \\
\frac{\partial^{3} \Psi_{n j}(0)}{\partial y^{3}}+k_{2} \Psi_{n j}(0)=0, & \frac{\partial^{3} \Psi_{n j}\left(L_{Y}\right)}{\partial y^{3}}+k_{2} \Psi_{n j}\left(L_{Y}\right)=0
\end{aligned}
$$

where $\mathrm{k}_{1}$ is the stiffness against rotation and $\mathrm{k}_{2}$ is the stiffness against translation.

Secondly, we consider an elastic rectangular plate resting on a variable Winkler elastic foundation and having elastic supports at all its edges, the boundary conditions are given by; Oni and Awodola (2010)

$$
\frac{\partial^{2} W(0, y, t)}{\partial x^{2}}-k_{1} \frac{\partial W(0, y, t)}{\partial x}=0, \quad \frac{\partial^{2} W\left(L_{X}, y, t\right)}{\partial x^{2}}-k_{1} \frac{\partial W\left(L_{X}, y, t\right)}{\partial x}=0
$$




$$
\begin{array}{ll}
\frac{\partial^{2} W(x, 0, t)}{\partial y^{2}}-k_{1} \frac{\partial W(x, 0, t)}{\partial y}=0, & \frac{\partial^{2} W\left(x, L_{Y}, t\right)}{\partial y^{2}}-k_{1} \frac{\partial W\left(x, L_{Y}, t\right)}{\partial y}=0 \\
\frac{\partial^{3} W(0, y, t)}{\partial x^{3}}+k_{2} W(0, y, t)=0, & \frac{\partial^{3} W\left(L_{X}, y, t\right)}{\partial x^{3}}+k_{2} W\left(L_{X}, y, t\right)=0 \\
\frac{\partial^{3} W(x, 0, t)}{\partial y^{3}}+k_{2} W(x, 0, t)=0, & \frac{\partial^{3} W\left(x, L_{Y}, t\right)}{\partial y^{3}}+k_{2} W\left(x, L_{Y}, t\right)=0
\end{array}
$$

and for normal modes

$$
\begin{array}{rlrl}
\frac{\partial^{2} \Psi_{n i}(0)}{\partial x^{2}}-k_{1} \frac{\partial \Psi_{n i}(0)}{\partial x}=0, & \frac{\partial^{2} \Psi_{n i}\left(L_{Y}\right)}{\partial x^{2}}-k_{1} \frac{\partial \Psi_{n i}\left(L_{Y}\right)}{\partial x}=0 \\
\frac{\partial^{2} \Psi_{n j}(0)}{\partial y^{2}}-k_{1} \frac{\partial \Psi_{n j}(0)}{\partial y}=0, & \frac{\partial^{2} \Psi_{n j}\left(L_{Y}\right)}{\partial y^{2}}-k_{1} \frac{\partial \Psi_{n j}\left(L_{Y}\right)}{\partial y}=0 \\
\frac{\partial^{3} \Psi_{n i}(0)}{\partial x^{3}}+k_{2} \Psi_{n i}(0)=0, & \frac{\partial^{3} \Psi_{n i}\left(L_{Y}\right)}{\partial x^{3}}+k_{2} \Psi_{n i}\left(L_{Y}\right)=0 \\
\frac{\partial^{3} \Psi_{n j}(0)}{\partial y^{3}}+k_{2} \Psi_{n j}(0) & =0, & \frac{\partial^{3} \Psi_{n j}\left(L_{Y}\right)}{\partial y^{3}}+k_{2} \Psi_{n j}\left(L_{Y}\right)=0
\end{array}
$$

where $\mathrm{k}_{1}$ and $\mathrm{k}_{2}$ are the stiffness against rotation and the stiffness against translation respectively.

\section{ANALYTICAL APPROXIMATE SOLUTION}

The method of analysis involves expressing the Dirac - Delta function as a Fourier cosine series. Because of the variable foundation term, the elegant method of the generalized integral transform breaks down while the generalized Galerkin's method used in one-dimensional structural problems (Beam problems) could not handle the two-dimensional structural problem (Plate problems). Thus, In order to solve equation (1), in the first instance, the deflection is written in the form; Shadnam et al (2001)

$$
W(x, y, t)=\sum_{n=1}^{\infty} \varphi_{n}(x, y) T_{n}(t)
$$


where $\phi_{\mathrm{n}}$ are the known eigenfunctions of the plate with the same boundary conditions. The $\phi_{\mathrm{n}}$ have the form of

$$
\nabla^{4} \varphi_{n}-\omega_{n}^{4} \varphi_{n}=0
$$

where

$$
\omega_{n}^{4}=\frac{\Omega_{n}^{2} \mu}{D}
$$

$\Omega_{\mathrm{n}}, \mathrm{n}=1,2,3, \ldots$, are the natural frequencies of the dynamical system and $\mathrm{T}_{\mathrm{n}}(\mathrm{t})$ are amplitude functions which have to be calculated.

At this juncture, the right hand side of equation (1) is written in the form of a series and we have

$$
\begin{aligned}
& R_{0}\left[\frac{\partial^{4}}{\partial t^{2} \partial x^{2}}+\frac{\partial^{4}}{\partial t^{2} \partial y^{2}}\right] W(x, y, t)-\frac{F_{0}}{\mu}\left[4 x-3 x^{2}+x^{3}\right] W(x, y, t)+\sum_{i=1}^{N}\left[\frac{M_{i} g}{\mu} \delta\left(x-c_{i} t\right) \delta(y-s)\right. \\
& \left.-\frac{M_{i}}{\mu}\left(\frac{\partial^{2}}{\partial t^{2}}+2 c_{i} \frac{\partial^{2}}{\partial t \partial x}+c_{i}^{2} \frac{\partial^{2}}{\partial x^{2}}\right) W(x, y, t) \delta\left(x-c_{i} t\right) \delta(y-s)\right]=\sum_{n=1}^{\infty} \varphi_{n}(x, y) B_{n}(t)
\end{aligned}
$$

Substituting equation (20) into equation (23) we have

$$
\begin{aligned}
& \sum_{n=1}^{\infty}\left\{R_{0}\left[\varphi_{n, x x}(x, y) T_{n, t t}(t)+\varphi_{n, y y}(x, y) T_{n, t t}(t)\right]-\frac{F_{0}}{\mu}\left[4 x-3 x^{2}+x^{3}\right] \varphi_{n}(x, y) T_{n}(t)\right. \\
& +\sum_{i=1}^{N}\left[\frac{M_{i} g}{\mu} \delta\left(x-c_{i} t\right) \delta(y-s)-\frac{M_{i}}{\mu}\left(\varphi_{n}(x, y) T_{n, t t}(t)+2 c_{i} \varphi_{n, x}(x, y) T_{n, t}(t)\right.\right. \\
& \left.\left.\left.+c_{i}^{2} \varphi_{n, x x}(x, y) T_{n}(t)\right) \delta\left(x-c_{i} t\right) \delta(y-s)\right]\right\}=\sum_{n=1}^{\infty} \varphi_{n}(x, y) B_{n}(t)
\end{aligned}
$$

where

$$
\begin{aligned}
& \varphi_{n, x}(x, y) \text { implies } \frac{\partial \varphi_{n}(x, y)}{\partial x}, \varphi_{n, x x}(x, y) \text { implies } \frac{\partial^{2} \varphi_{n}(x, y)}{\partial x^{2}}, \\
& \varphi_{n, y}(x, y) \text { implies } \frac{\partial \varphi_{n}(x, y)}{\partial y}, \varphi_{n, y y}(x, y) \text { implies } \frac{\partial^{2} \varphi_{n}(x, y)}{\partial y^{2}}, \\
& T_{n, t}(t) \text { implies } \frac{d T_{n}(t)}{d t} \text { and } T_{n, t t}(t) \text { implies } \frac{d^{2} T_{n}(t)}{d t^{2}}
\end{aligned}
$$


Multiplying both sides of equation (24) by $\phi_{\mathrm{p}}(\mathrm{x}, \mathrm{y})$ and integrating on area $\mathrm{A}$ of the plate, we have

$$
\begin{aligned}
& \sum_{n=1}^{\infty} \int_{A}\left\{R_{0}\left[\varphi_{n, x x}(x, y) \varphi_{p}(x, y) T_{n, t t}(t)+\varphi_{n, y y}(x, y) \varphi_{p}(x, y) T_{n, t t}(t)\right]\right. \\
& -\frac{F_{0}}{\mu}\left[4 x-3 x^{2}+x^{3}\right] \varphi_{n}(x, y) \varphi_{p}(x, y) T_{n}(t)+\sum_{i=1}^{N}\left[\frac{M_{i} g}{\mu} \varphi_{p}(x, y) \delta\left(x-c_{i} t\right) \delta(y-s)\right. \\
& -\frac{M_{i}}{\mu}\left(\varphi_{n}(x, y) \varphi_{p}(x, y) T_{n, t t}(t)+2 c_{i} \varphi_{n, x}(x, y) \varphi_{p}(x, y) T_{n, t}(t)\right. \\
& \left.\left.\left.+c_{i}^{2} \varphi_{n, x x}(x, y) \varphi_{p}(x, y) T_{n}(t)\right) \delta\left(x-c_{i} t\right) \delta(y-s)\right]\right\} d A=\sum_{n=1}^{\infty} \int_{A} \varphi_{n}(x, y) \varphi_{p}(x, y) B_{n}(t) d A
\end{aligned}
$$

Considering the orthogonality of $\phi_{\mathrm{n}}(\mathrm{x}, \mathrm{y})$

$$
\begin{aligned}
B_{n}(t) & =\frac{1}{P^{*}} \sum_{n=1}^{\infty} \int_{A}\left\{R_{0}\left[\varphi_{n, x x}(x, y) \varphi_{p}(x, y) T_{n, t t}(t)+\varphi_{n, y y}(x, y) \varphi_{p}(x, y) T_{n, t t}(t)\right]\right. \\
& -\frac{F_{0}}{\mu}\left[4 x-3 x^{2}+x^{3}\right] \varphi_{n}(x, y) \varphi_{p}(x, y) T_{n}(t)+\sum_{i=1}^{N}\left[\frac{M_{i} g}{\mu} \varphi_{p}(x, y) \delta\left(x-c_{i} t\right) \delta(y-s)\right. \\
& -\frac{M_{i}}{\mu}\left(\varphi_{n}(x, y) \varphi_{p}(x, y) T_{n, t t}(t)+2 c_{i} \varphi_{n, x}(x, y) \varphi_{p}(x, y) T_{n, t}(t)\right. \\
& \left.\left.\left.+c_{i}^{2} \varphi_{n, x x}(x, y) \varphi_{p}(x, y) T_{n}(t)\right) \delta\left(x-c_{i} t\right) \delta(y-s)\right]\right\} d A
\end{aligned}
$$

where

$$
P^{*}=\int_{A} \varphi_{p}^{2} d A
$$

Using (27), equation (1), taken into account (21), can be written as

$$
\begin{aligned}
& \varphi_{n}(x, y)\left[\frac{D \omega_{n}^{4}}{\mu} T_{n}(t)+T_{n, t t}(t)\right]=\frac{\varphi_{n}(x, y)}{P^{*}} \sum_{q=1}^{\infty} \int_{A}\left\{R _ { 0 } \left[\varphi_{q, x x}(x, y) \varphi_{p}(x, y) T_{q, t t}(t)\right.\right. \\
& \left.+\varphi_{q, y y}(x, y) \varphi_{p}(x, y) T_{q, t t}(t)\right]-\frac{F_{0}}{\mu}\left[4 x-3 x^{2}+x^{3}\right] \varphi_{q}(x, y) \varphi_{p}(x, y) T_{q}(t) \\
& +\sum_{i=1}^{N}\left[\frac{M_{i} g}{\mu} \varphi_{p}(x, y) \delta\left(x-c_{i} t\right) \delta(y-s)-\frac{M_{i}}{\mu}\left(\varphi_{q}(x, y) \varphi_{p}(x, y) T_{q, t t}(t)\right.\right. \\
& \left.\left.\left.+2 c_{i} \varphi_{q, x}(x, y) \varphi_{p}(x, y) T_{q, t}(t)+c_{i}^{2} \varphi_{q, x x}(x, y) \varphi_{p}(x, y) T_{q}(t)\right) \delta\left(x-c_{i} t\right) \delta(y-s)\right]\right\} d A
\end{aligned}
$$


Equation (28) must be satisfied for arbitrary x, y (that is, each point of the plate) and this is possible only when

$$
\begin{aligned}
& T_{n, t t}(t)+\frac{D \omega_{n}^{4}}{\mu} T_{n}(t)=\frac{1}{P^{*}} \sum_{q=1}^{\infty} \int_{A}\left\{R _ { 0 } \left[\varphi_{q, x x}(x, y) \varphi_{p}(x, y) T_{q, t t}(t)\right.\right. \\
& \left.+\varphi_{q, y y}(x, y) \varphi_{p}(x, y) T_{q, t t}(t)\right]-\frac{F_{0}}{\mu}\left[4 x-3 x^{2}+x^{3}\right] \varphi_{q}(x, y) \varphi_{p}(x, y) T_{q}(t) \\
& +\sum_{i=1}^{N}\left[\frac{M_{i} g}{\mu} \varphi_{p}(x, y) \delta\left(x-c_{i} t\right) \delta(y-s)-\frac{M_{i}}{\mu}\left(\varphi_{q}(x, y) \varphi_{p}(x, y) T_{q, t t}(t)\right.\right. \\
& \left.\left.\left.+2 c_{i} \varphi_{q, x}(x, y) \varphi_{p}(x, y) T_{q, t}(t)+c_{i}^{2} \varphi_{q, x x}(x, y) \varphi_{p}(x, y) T_{q}(t)\right) \delta\left(x-c_{i} t\right) \delta(y-s)\right]\right\} d A
\end{aligned}
$$

The system in equation (29) is a set of coupled ordinary differential equations.

Considering the property of the Dirac-Delta function and expressing it in the Fourier cosine series as

$$
\delta\left(x-c_{i} t\right)=\frac{1}{L_{X}}\left[1+2 \sum_{j=1}^{\infty} \cos \frac{j \pi c_{i} t}{L_{X}} \cos \frac{j \pi x}{L_{X}}\right]
$$

and

$$
\delta(y-s)=\frac{1}{L_{Y}}\left[1+2 \sum_{k=1}^{\infty} \cos \frac{k \pi s}{L_{Y}} \cos \frac{k \pi y}{L_{Y}}\right]
$$

equation (29) becomes

$$
\begin{aligned}
& \frac{d^{2} T_{n}(t)}{d t^{2}}+\alpha_{n}^{2} T_{n}(t)-\frac{1}{P^{*}} \sum_{q=1}^{\infty}\left\{R_{0} P_{1}^{*} \frac{d^{2} T_{q}(t)}{d t^{2}}-\frac{F_{0}}{\mu} P_{2}^{*} T_{q}(t)\right. \\
& -\sum_{i=1}^{N} \frac{M_{i}}{L_{X} L_{Y} \mu}\left[2 \left(\frac{P_{3}^{*}}{2}+\sum_{k=1}^{\infty} \cos \frac{k \pi s}{L_{Y}} P_{3}^{* * *}(k)+\sum_{j=1}^{\infty} \cos \frac{j \pi c_{i} t}{L_{X}} P_{3}^{* * * *}(j)\right.\right. \\
& \left.+2 \sum_{j=1}^{\infty} \sum_{k=1}^{\infty} \cos \frac{j \pi c_{i} t}{L_{X}} \cos \frac{k \pi s}{L_{Y}} P_{3}^{* * * * * *}(j, k)\right) \frac{d^{2} T_{q}(t)}{d t^{2}}+4 c_{i}\left(\frac{P_{4}^{*}}{2}+\sum_{k=1}^{\infty} \cos \frac{k \pi s}{L_{Y}} P_{4}^{* *}(k)\right.
\end{aligned}
$$




$$
\begin{aligned}
& \left.+\sum_{j=1}^{\infty} \cos \frac{j \pi c_{i} t}{L_{X}} P_{4}^{* * * * *}(j)+2 \sum_{j=1}^{\infty} \sum_{k=1}^{\infty} \cos \frac{j \pi c_{i} t}{L_{X}} \cos \frac{k \pi s}{L_{Y}} P_{4}^{* * *+*}(j, k)\right) \frac{d T_{q}(t)}{d t} \\
& +2 c_{i}^{2}\left(\frac{P_{5}^{*}}{2}+\sum_{k=1}^{\infty} \cos \frac{k \pi s}{L_{Y}} P_{5}^{* * *}(k)+\sum_{j=1}^{\infty} \cos \frac{j \pi c_{i} t}{L_{X}} P_{5}^{* * * * *}(j)\right. \\
& \left.\left.\left.+2 \sum_{j=1}^{\infty} \sum_{k=1}^{\infty} \cos \frac{j \pi c_{i} t}{L_{X}} \cos \frac{k \pi s}{L_{Y}} P_{5}^{* * * * * *}(j, k)\right) T_{q}(t)\right]\right\}=\sum_{i=1}^{N} \frac{M_{i} g}{P^{*} \mu} \varphi_{p}\left(c_{i} t, s\right)
\end{aligned}
$$

where $\alpha_{n}^{2}=\frac{D \omega_{n}^{4}}{\mu}$

$P_{1}^{*}=\int_{0}^{L_{X}} \int_{0}^{L_{Y}}\left[\varphi_{n, x x}(x, y)+\varphi_{n, y y}(x, y)\right] \varphi_{p}(x, y) d y d x, P_{2}^{*}=\int_{0}^{L_{X}} \int_{0}^{L_{Y}}\left[4 x-3 x^{2}+x^{3}\right] \varphi_{n}(x, y) \varphi_{p}(x, y) d y d x$

$P_{3}^{*}=\int_{0}^{L_{X}} \int_{0}^{L_{Y}} \phi_{n}(x, y) \phi_{p}(x, y) d y d x, \quad P_{3}^{* *}(k)=\int_{0}^{L_{X}} \int_{0}^{L_{Y}} \cos \frac{k \pi y}{L_{Y}} \phi_{n}(x, y) \phi_{p}(x, y) d y d x$

$P_{3}^{* * * *}(j)=\int_{0}^{L_{X}} \int_{0}^{L_{Y}} \cos \frac{j \pi x}{L_{X}} \varphi_{n}(x, y) \varphi_{p}(x, y) d y d x, P_{3}^{* * * * *}(j, k)=\int_{0}^{L_{X}} \int_{0}^{L_{Y}} \cos \frac{j \pi x}{L_{X}} \cos \frac{k \pi y}{L_{Y}} \varphi_{n}(x, y) \varphi_{p}(x, y) d y d x$,

$P_{4}^{*}=\int_{0}^{L_{X}} \int_{0}^{L_{Y}} \varphi_{n, x}(x, y) \varphi_{p}(x, y) d y d x, \quad P_{4}^{* *}(k)=\int_{0}^{L_{X}} \int_{0}^{L_{Y}} \cos \frac{k \pi y}{L_{Y}} \varphi_{n, x}(x, y) \varphi_{p}(x, y) d y d x$,

$P_{4}^{* * * *}(j)=\int_{0}^{L_{X}} \int_{0}^{L_{Y}} \cos \frac{j \pi x}{L_{X}} \varphi_{n, x}(x, y) \varphi_{p}(x, y) d y d x, P_{4}^{* * * * *}(j, k)=\int_{0}^{L_{X}} \int_{0}^{L_{Y}} \cos \frac{j \pi x}{L_{X}} \cos \frac{k \pi y}{L_{Y}} \varphi_{n, x}(x, y) \varphi_{p}(x, y) d y d x$,

$P_{5}^{*}=\int_{0}^{L_{X}} \int_{0}^{L_{Y}} \varphi_{n, x x}(x, y) \varphi_{p}(x, y) d y d x, P_{5}^{* * *}(k)=\int_{0}^{L_{X}} \int_{0}^{L_{Y}} \cos \frac{k \pi y}{L_{Y}} \varphi_{n, x x}(x, y) \varphi_{p}(x, y) d y d x$,

$P_{5}^{* * * *}(j)=\int_{0}^{L_{X}} \int_{0}^{L_{Y}} \cos \frac{j \pi x}{L_{X}} \varphi_{n, x x}(x, y) \varphi_{p}(x, y) d y d x$ and $P_{5}^{* * * * * *}(j, k)=\int_{0}^{L_{X}} \int_{0}^{L_{Y}} \cos \frac{j \pi x}{L_{X}} \cos \frac{k \pi y}{L_{Y}} \varphi_{n, x x}(x, y) \varphi_{p}(x, y) d y d x$,

Equation (32) is the transformed equation governing the problem of an elastically supported rectangular plate on a variable Winkler elastic foundation. This is a coupled second order differential equation.

In what follows, $\phi_{\mathrm{n}}(\mathrm{x}, \mathrm{y})$ are assumed to be the products of the functions $\psi_{\mathrm{ni}}(\mathrm{x})$ and $\psi_{\mathrm{nj}}(\mathrm{y})$ which are the beam functions in the directions of $\mathrm{x}$ and $\mathrm{y}$ axes respectively, Lee and $\mathrm{Ng}$ (1996). That is

$$
\varphi_{n}(x, y)=\psi_{n i}(x) \psi_{n j}(y)
$$


Since each of these beam functions satisfies the boundary conditions in its direction, the kernel (the product of these beam functions) in the above integrals satisfies all boundary conditions for any plate problem of practical interest. In particular, these beam functions can be defined respectively, as

$$
\psi_{n i}(x)=\sin \frac{\Omega_{n i} x}{L_{X}}+A_{n i} \cos \frac{\Omega_{n i} x}{L_{X}}+B_{n i} \sinh \frac{\Omega_{n i} x}{L_{X}}+C_{n i} \cosh \frac{\Omega_{n i} x}{L_{X}}
$$

and

$$
\psi_{n j}(x)=\sin \frac{\Omega_{n j} y}{L_{Y}}+A_{n j} \cos \frac{\Omega_{n j} y}{L_{Y}}+B_{n j} \sinh \frac{\Omega_{n j} y}{L_{Y}}+C_{n j} \cosh \frac{\Omega_{n j} y}{L_{Y}}
$$

where $\mathrm{A}_{\mathrm{ni}}, \mathrm{A}_{\mathrm{nj}}, \mathrm{B}_{\mathrm{ni}}, \mathrm{B}_{\mathrm{nj}}, \mathrm{C}_{\mathrm{ni}}$ and $\mathrm{C}_{\mathrm{nj}}$ are constants determined by the boundary conditions. $\Omega_{\mathrm{ni}}$ and $\Omega_{\mathrm{nj}}$ are called the mode frequencies.

In order to solve equation (32) we shall consider a mass $\mathrm{M}$ traveling with constant velocity c along the line $\mathrm{y}=\mathrm{s}$. The solution for any arbitrary number of moving masses can be obtained by superposition of the individual solution since the governing differential equation is linear. Thus for the single mass $\mathrm{M}_{1}$ equation (32) reduces to

$$
\begin{aligned}
& \frac{d^{2} T_{n}(t)}{d t^{2}}+\alpha_{n}^{2} T_{n}(t)-\frac{1}{P^{*}} \sum_{q=1}^{\infty}\left\{R_{0} P_{1}^{*} \frac{d^{2} T_{q}(t)}{d t^{2}}-\frac{F_{0}}{\mu} P_{2}^{*} T_{q}(t)\right. \\
& -\Gamma\left[2 \left(\frac{P_{3}^{*}}{2}+\sum_{k=1}^{\infty} \cos \frac{k \pi s}{L_{Y}} P_{3}^{* *}(k)+\sum_{j=1}^{\infty} \cos \frac{j \pi c t}{L_{X}} P_{3}^{* * *}(j)\right.\right. \\
& +2 \sum_{j=1}^{\infty} \sum_{k=1}^{\infty} \cos \frac{j \pi c t}{L_{X}} \cos \frac{k \pi s}{L_{Y}} P_{3}^{* * * *}(j, k) \frac{d^{2} T_{q}(t)}{d t^{2}}+4 c\left(\frac{P_{4}^{*}}{2}+\sum_{k=1}^{\infty} \cos \frac{k \pi s}{L_{Y}} P_{4}^{* * *}(k)\right. \\
& \left.+\sum_{j=1}^{\infty} \cos \frac{j \pi c t}{L_{X}} P_{4}^{* * *}(j)+2 \sum_{j=1}^{\infty} \sum_{k=1}^{\infty} \cos \frac{j \pi c t}{L_{X}} \cos \frac{k \pi s}{L_{Y}} P_{4}^{* * * * *}(j, k)\right) \frac{d T_{q}(t)}{d t} \\
& +2 c^{2}\left(\frac{P_{5}^{*}}{2}+\sum_{k=1}^{\infty} \cos \frac{k \pi s}{L_{Y}} P_{5}^{* *}(k)+\sum_{j=1}^{\infty} \cos \frac{j \pi c t}{L_{X}} P_{5}^{* * *}(j)\right. \\
& \left.\left.\left.+2 \sum_{j=1}^{\infty} \sum_{k=1}^{\infty} \cos \frac{j \pi c t}{L_{X}} \cos \frac{k \pi s}{L_{Y}} P_{5}^{* * * *}(j, k)\right) T_{q}(t)\right]\right\}=\frac{M g}{P^{*} \mu} \Psi_{p i}(c t) \Psi_{p j}(s)
\end{aligned}
$$

where 


$$
\Gamma=\frac{M}{L_{X} L_{Y} \mu}
$$

Equation (36) is the fundamental equation of our problem. In what follows, we shall discuss two special cases of the equation (36) namely; the moving force and the moving mass problems.

\section{CASE I: RECTANGULAR PLATE TRAVERSED BY A MOVING FORCE}

Setting $\Gamma=0$ in equation (36) gives an approximate model of the differential equation describing the response of a rectangular plate resting on a variable Winkler elastic foundation and traversed by a moving force. Thus, if $\Gamma=0$ in equation (36), we have

$$
\frac{d^{2} T_{n}(t)}{d t^{2}}+\alpha_{n}^{2} T_{n}(t)-\frac{P_{1}^{*} R_{0}}{P^{*}} \sum_{q=1}^{\infty} \frac{d^{2} T_{q}(t)}{d t^{2}}+\frac{P_{2}^{*} F_{0}}{\mu P^{*}} \sum_{q=1}^{\infty} T_{q}(t)=\frac{M g}{P^{*} \mu} \Psi_{p i}(c t) \Psi_{p j}(s)
$$

Evidently, an exact analytical solution to this equation is not possible. Consequently, the approximate analytical solution technique, which is a modification of the asymptotic method of Struble discussed in Gbadeyan and Oni (1995) shall be used.

To solve equation (38), first, we neglect the rotatory inertial term and rearrange the equation to take the form

$$
\frac{d^{2} T_{n}(t)}{d t^{2}}+\left[\alpha_{n}^{2}+\Gamma^{*} P_{2}^{*}\right] T_{n}(t)+\Gamma^{*} P_{2}^{*} \sum_{\substack{q=1 \\ q \neq n}}^{\infty} T_{q}(t)=\frac{M g}{P^{*} \mu} \Psi_{p i}(c t) \Psi_{p j}(s)
$$

where

$$
\Gamma^{*}=\frac{F_{0}}{\mu P^{*}}
$$

Consider a parameter $\lambda<1$ for any arbitrary ratio $\Gamma^{*}$ defined as

$$
\lambda=\frac{\Gamma^{*}}{1+\Gamma^{*}}
$$

so that

$$
\Gamma^{*}=\lambda+o\left(\lambda^{2}\right)
$$


Substituting equation (42) into the homogenous part of equation (39) yields

$$
\frac{d^{2} T_{n}(t)}{d t^{2}}+\left[\alpha_{n}^{2}+\lambda P_{2}^{*}\right] T_{n}(t)+\lambda P_{2}^{*} \sum_{\substack{q=1 \\ q \neq n}}^{\infty} T_{q}(t)=0
$$

When $\lambda$ is set to zero in equation (43), a situation corresponding to the case in which the effect of the foundation is regarded as negligible is obtained.

Struble's technique requires that the asymptotic solution of the homogenous part of equation (39) be of the form

$$
T_{n}(t)=A_{n}(t) \cos \left[\alpha_{n} t-\Phi_{n}(t)\right]+\lambda T_{1}(t)+o\left(\lambda^{2}\right)
$$

where $\mathrm{A}_{\mathrm{n}}(\mathrm{t})$ and $\Phi_{\mathrm{n}}(\mathrm{t})$ are slowly varying functions of time or equivalently

$$
\begin{aligned}
\frac{d A_{n}(t)}{d t} & \rightarrow o(\lambda) ; & \frac{d^{2} A_{n}(t)}{d t^{2}} & \rightarrow 0\left(\lambda^{2}\right) \\
\frac{d \Phi_{n}(t)}{d t} & \rightarrow o(\lambda) ; & \frac{d^{2} \Phi_{n}(t)}{d t^{2}} & \rightarrow 0\left(\lambda^{2}\right)
\end{aligned}
$$

where $\rightarrow$ implies " is of "

Thus, equation (43) can be replaced with

$$
\frac{d^{2} T_{n}(t)}{d t^{2}}+\gamma_{s}^{2} T_{n}(t)=0
$$

where

$$
\gamma_{s}=\alpha_{n}+\frac{\lambda P_{2}^{*}}{2 \alpha_{n}}
$$

represents the modified frequency due to the effect of the foundation. It is observed that when $\lambda$ $=0$, we recover the frequency of the moving force problem when the effect of the foundation is neglected.

Thus; using (47), equation (38) can be written as

$$
\frac{d^{2} T_{n}(t)}{d t^{2}}+\gamma_{s}^{2} T_{n}(t)-\frac{P_{1}^{*} R_{0}}{P^{*}} \sum_{q=1}^{\infty} \frac{d^{2} T_{q}(t)}{d t^{2}}=\frac{M g}{P^{*} \mu} \Psi_{p i}(c t) \Psi_{p j}(s)
$$


The homogenous part of equation (48) is rearranged to take the form

$$
\frac{d^{2} T_{n}(t)}{d t^{2}}+\frac{\gamma_{s}^{2}}{1-\lambda_{0} P_{1}^{*}} T_{n}(t)-\frac{\lambda_{0} P_{1}^{*}}{1-\lambda_{0} P_{1}^{*}} \sum_{\substack{q=1 \\ q \neq n}}^{\infty} \frac{d^{2} T_{q}(t)}{d t^{2}}=0
$$

where $\quad \lambda_{0}=\frac{R_{0}}{P^{*}}$

Now consider the parameter $\varepsilon_{0}<1$ for any arbitrary mass ratio $\lambda_{0}$ defined as

$$
\varepsilon_{0}=\frac{\lambda_{0}}{1+\lambda_{0}}
$$

It can be shown that

$$
\lambda_{0}=\varepsilon_{0}+o\left(\varepsilon_{0}^{2}\right)
$$

Following the same argument, equation (49) can be replaced with

$$
\frac{d^{2} T_{n}(t)}{d t^{2}}+\gamma_{s f}^{2} T_{n}(t)=0
$$

where

$$
\gamma_{s f}=\gamma_{s}\left[1+\frac{\varepsilon_{0} P_{1}^{*}}{2}\right]
$$

is the modified frequency corresponding to the frequency of the free system due to the presence of the rotatory inertia. It is observed that when $\varepsilon_{0}=0$, we recover the frequency of the moving force problem when the rotatory inertia effect is neglected.

In order to solve the non-homogenous equation (48), the differential operator which acts on $\mathrm{T}_{\mathrm{n}}(\mathrm{t})$ is replaced by the equivalent free system operator defined by the modified frequency $\gamma_{\mathrm{sf}}$. Thus

$$
\frac{d^{2} T_{n}(t)}{d t^{2}}+\gamma_{s f}^{2} T_{n}(t)=K_{0} \Psi_{p i}(c t) \Psi_{p j}(s)
$$

where 


$$
K_{0}=\frac{M g}{P^{*} \mu}
$$

Therefore, the moving force problem is reduced to the non-homogeneous ordinary differential equation given as

$$
\frac{d^{2} T_{n}(t)}{d t^{2}}+\gamma_{s f}^{2} T_{n}(t)=K_{0} \Psi_{p j}(s)\left[\sin \alpha_{p i} t+A_{p i} \cos \alpha_{p i} t+B_{p i} \sinh \alpha_{p i} t+C_{p i} \cosh \alpha_{p i} t\right]
$$

where $\alpha_{p i}=\frac{\Omega_{p i} c}{L_{X}}$

When equation (56) is solved in conjunction with the initial conditions, one obtains expression for $T_{n}(t)$. Thus in view of equation (20), one obtains

$$
\begin{aligned}
W(x, y, t) & =\sum_{n i=1}^{\infty} \sum_{n j=1}^{\infty} \frac{K_{0} \Psi_{p j}(s)}{\gamma_{s f}\left[\gamma_{s f}^{4}-\alpha_{p i}^{4}\right]}\left\{[ \gamma _ { s f } ^ { 2 } - \alpha _ { p i } ^ { 2 } ] \left[C_{p i} \gamma_{s f}\left(\cosh \alpha_{p i} t-\cos \gamma_{s f} t\right)\right.\right. \\
& \left.+B_{p i}\left(\gamma_{s f} \sinh \alpha_{p i} t-\alpha_{p i} \sin \gamma_{s f} t\right)\right]+\left[\gamma_{s f}^{2}+\alpha_{p i}^{2}\right]\left[A_{p i} \gamma_{s f}\left(\cos \alpha_{p i} t-\cos \gamma_{s f} t\right)\right. \\
& \left.\left.-\left(\alpha_{p i} \sin \gamma_{s f} t-\gamma_{s f} \sin \alpha_{p i} t\right)\right]\right\}\left[\sin \frac{\Omega_{n i} x}{L_{X}}+A_{n i} \cos \frac{\Omega_{n i} x}{L_{X}}+B_{n i} \sinh \frac{\Omega_{n i} x}{L_{X}}\right. \\
& \left.+C_{n i} \cosh \frac{\Omega_{n i} x}{L_{X}}\right]\left[\sin \frac{\Omega_{n j} y}{L_{Y}}+A_{n j} \cos \frac{\Omega_{n j} y}{L_{Y}}+B_{n j} \sinh \frac{\Omega_{n j} y}{L_{Y}}+C_{n j} \cosh \frac{\Omega_{n j} y}{L_{Y}}\right] \text { (57) }
\end{aligned}
$$

Equation (57) represents the transverse displacement response to a moving force of a rectangular plate resting on variable Winkler elastic foundation.

\section{CASE II: RECTANGULAR PLATE TRAVERSED BY A MOVING MASS}

If the mass of the moving load is commensurable with that of the structure, the inertia effect of the moving mass is not negligible. Thus $\Gamma \neq 0$ and one is required to solve the entire equation (36) when no term of the coupled differential equation is neglected. This is termed the moving mass problem.

Thus, equation (36) can be rewritten in the form 


$$
\begin{aligned}
& {\left[1+\frac{2 \varepsilon}{P^{*}}\left(\frac{P_{3}^{*}}{2}+\sum_{k=1}^{\infty} \cos \frac{k \pi s}{L_{Y}} P_{3}^{* * *}(k)+\sum_{j=1}^{\infty} \cos \frac{j \pi c t}{L_{X}} P_{3}^{* * * *}(j)+2 \sum_{j=1}^{\infty} \sum_{k=1}^{\infty} \cos \frac{j \pi c t}{L_{X}} \cos \frac{k \pi s}{L_{Y}} P_{3}^{* * * * * *}(j, k)\right)\right] \frac{d^{2} T_{n}(t)}{d t^{2}}} \\
& +\frac{4 \varepsilon c}{P^{*}}\left(\frac{P_{4}^{*}}{2}+\sum_{k=1}^{\infty} \cos \frac{k \pi s}{L_{Y}} P_{4}^{* * *}(k)+\sum_{j=1}^{\infty} \cos \frac{j \pi c t}{L_{X}} P_{4}^{* * * *}(j)+2 \sum_{j=1}^{\infty} \sum_{k=1}^{\infty} \cos \frac{j \pi c t}{L_{X}} \cos \frac{k \pi s}{L_{Y}} P_{4}^{* * * * *}(j, k)\right) \frac{d T_{n}(t)}{d t} \\
& +\left[\gamma_{s f}^{2}+\frac{2 \varepsilon c^{2}}{P^{*}}\left(\frac{P_{5}^{*}}{2}+\sum_{k=1}^{\infty} \cos \frac{k \pi s}{L_{Y}} P_{5}^{* * *}(k)+\sum_{j=1}^{\infty} \cos \frac{j \pi c t}{L_{X}} P_{5}^{* * * *}(j)+2 \sum_{j=1}^{\infty} \sum_{k=1}^{\infty} \cos \frac{j \pi c t}{L_{X}} \cos \frac{k \pi s}{L_{Y}} P_{5}^{* * * * * *}(j, k)\right)\right] T_{n}(t) \\
& +\frac{\varepsilon}{P^{*}} \sum_{q=1}\left[2\left(\frac{P_{3}^{*}}{2}+\sum_{k=1}^{\infty} \cos \frac{k \pi s}{L_{Y}} P_{3}^{* *}(k)+\sum_{j=1}^{\infty} \cos \frac{j \pi c t}{L_{X}} P_{3}^{* * * *}(j)+2 \sum_{j=1}^{\infty} \sum_{k=1}^{\infty} \cos \frac{j \pi c t}{L_{X}} \cos \frac{k \pi s}{L_{Y}} P_{3}^{* * * * * *}(j, k)\right) \frac{d^{2} T_{q}(t)}{d t^{2}}\right. \\
& 4 c\left(\frac{P_{4}^{*}}{2}+\sum_{k=1}^{\infty} \cos \frac{k \pi s}{L_{Y}} P_{4}^{* * *}(k)+\sum_{j=1}^{\infty} \cos \frac{j \pi c t}{L_{X}} P_{4}^{* * * *}(j)+2 \sum_{j=1}^{\infty} \sum_{k=1}^{\infty} \cos \frac{j \pi c t}{L_{X}} \cos \frac{k \pi s}{L_{Y}} P_{4}^{* * * * *}(j, k)\right) \frac{d T_{q}(t)}{d t} \\
& \left.+2 c^{2}\left(\frac{P_{5}^{*}}{2}+\sum_{k=1}^{\infty} \cos \frac{k \pi s}{L_{Y}} P_{5}^{* * *}(k)+\sum_{j=1}^{\infty} \cos \frac{j \pi c t}{L_{X}} P_{5}^{* * * * *}(j)+2 \sum_{j=1}^{\infty} \sum_{k=1}^{\infty} \cos \frac{j \pi c t}{L_{X}} \cos \frac{k \pi s}{L_{Y}} P_{5}^{* * * * * k}(j, k)\right) T_{q}(t)\right] \\
& =\frac{\varepsilon g L_{X} L_{Y}}{P^{*}} \Psi_{p i}(c t) \Psi_{p j}(s)
\end{aligned}
$$

where $\varepsilon=\frac{M}{L_{X} L_{Y} \mu}$

we rearrange equation (58) to take the form

$$
\begin{aligned}
& \frac{d^{2} T_{n}(t)}{d t^{2}}+\frac{\mu_{0} R_{2}(t)}{1+\mu_{0} R_{1}(t)} \frac{d T_{n}(t)}{d t}+\frac{\gamma_{s f}^{2}+\mu_{0} R_{3}(t)}{1+\mu_{0} R_{1}(t)} T_{n}(t)+\frac{\mu_{0}}{1+\mu_{0} R_{1}(t)} \sum_{\substack{q=1 \\
q \neq n}}^{\infty}\left[R_{1}(t) \frac{d^{2} T_{q}(t)}{d t^{2}}+R_{2}(t) \frac{d T_{q}(t)}{d t}\right. \\
& \left.+R_{3}(t) T_{q}(t)\right]=\frac{\mu_{0} g L_{X} L_{Y}}{\left[1+\mu_{0} R_{1}(t)\right] P^{*}} \Psi_{p i}(c t) \Psi_{p j}(s)
\end{aligned}
$$

where $\varepsilon$ has been written as a function of the mass ratio $\mu_{\mathrm{o}}$,

$$
\begin{aligned}
& R_{1}(t)=\frac{2}{P^{*}}\left[\frac{P_{3}^{*}}{2}+\sum_{k=1}^{\infty} \cos \frac{k \pi s}{L_{Y}} P_{3}^{* * *}(k)+\sum_{j=1}^{\infty} \cos \frac{j \pi c t}{L_{X}} P_{3}^{* * * * *}(j)+2 \sum_{j=1}^{\infty} \sum_{k=1}^{\infty} \cos \frac{j \pi c t}{L_{X}} \cos \frac{k \pi s}{L_{Y}} P_{3}^{* * * * *}(j, k)\right] \\
& R_{2}(t)=\frac{2 c}{P^{*}}\left[\frac{P_{4}^{*}}{2}+\sum_{k=1}^{\infty} \cos \frac{k \pi s}{L_{Y}} P_{4}^{* * *}(k)+\sum_{j=1}^{\infty} \cos \frac{j \pi c t}{L_{X}} P_{4}^{* * * *}(j)+2 \sum_{j=1}^{\infty} \sum_{k=1}^{\infty} \cos \frac{j \pi c t}{L_{X}} \cos \frac{k \pi s}{L_{Y}} P_{4}^{* * * * *}(j, k)\right] \\
& R_{3}(t)=\frac{2 c^{2}}{P^{*}}\left[\frac{P_{5}^{*}}{2}+\sum_{k=1}^{\infty} \cos \frac{k \pi s}{L_{Y}} P_{5}^{* * *}(k)+\sum_{j=1}^{\infty} \cos \frac{j \pi c t}{L_{X}} P_{5}^{* * * * *}(j)+2 \sum_{j=1}^{\infty} \sum_{k=1}^{\infty} \cos \frac{j \pi c t}{L_{X}} \cos \frac{k \pi s}{L_{Y}} P_{5}^{* * * * * *}(j, k)\right]
\end{aligned}
$$


Considering the homogeneous part of the equation (59) and going through the same arguments and analysis as in the previous case, the modified frequency corresponding to the frequency of the free system due to the presence of the moving mass is

$$
\beta_{s f}=\gamma_{s f}\left[1-\frac{\mu_{0}}{2}\left(R_{1}-\frac{R_{3}}{\gamma_{s f}^{2}}\right)\right]
$$

retaining terms to o $\left(\mu_{\mathrm{o}}\right)$ only.

Thus, to solve the non-homogeneous equation (59), the differential operator which acts on $\mathrm{T}_{\mathrm{n}}(\mathrm{t})$ and $\mathrm{T}_{\mathrm{q}}(\mathrm{t})$ is replaced by the equivalent free system operator defined by the modified fre-

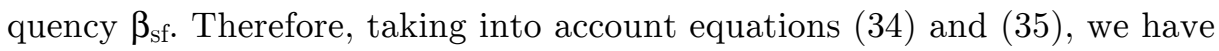

$$
\frac{d^{2} T_{n}(t)}{d t^{2}}+\beta_{s f}^{2} T_{n}(t)=G_{0} \Psi_{p j}(s) \sin \alpha_{p i} t+A_{p i} \cos \alpha_{p i} t+B_{p i} \sinh \alpha_{p i} t+C_{p i} \cosh \alpha_{p i} t
$$

where

$$
G_{0}=\frac{\mu_{0} g L_{X} L_{Y}}{P^{*}}
$$

It is noticed that equation (61) is analogous to equation (56) with $\beta_{\mathrm{sf}}$ and $\mathrm{G}_{0}$ replacing $\gamma_{\mathrm{sf}}$ and $\mathrm{K}_{0}$ respectively. Therefore, when equation (61) is solved in conjunction with the initial conditions, one obtains expression for $\mathrm{T}_{\mathrm{n}}(\mathrm{t})$ and in view of equation (20), one obtains

$$
\begin{aligned}
W(x, y, t) & =\sum_{n i=1}^{\infty} \sum_{n j=1}^{\infty} \frac{G_{0} \Psi_{p j}(s)}{\beta_{s f}\left[\beta_{s f}^{4}-\alpha_{p i}^{4}\right]}\left\{[ \beta _ { s f } ^ { 2 } - \alpha _ { p i } ^ { 2 } ] \left[C_{p i} \beta_{s f}\left(\cosh \alpha_{p i} t-\cos \beta_{s f} t\right)\right.\right. \\
& \left.+B_{p i}\left(\beta_{s f} \sinh \alpha_{p i} t-\alpha_{p i} \sin \beta_{s f} t\right)\right]+\left[\beta_{s f}^{2}+\alpha_{p i}^{2}\right]\left[A_{p i} \beta_{s f}\left(\cos \alpha_{p i} t-\cos \beta_{s f} t\right)\right. \\
& \left.\left.-\left(\alpha_{p i} \sin \beta_{s f} t-\beta_{s f} \sin \alpha_{p i} t\right)\right]\right\}\left[\sin \frac{\Omega_{n i} x}{L_{X}}+A_{n i} \cos \frac{\Omega_{n i} x}{L_{X}}+B_{n i} \sinh \frac{\Omega_{n i} x}{L_{X}}\right. \\
& \left.+C_{n i} \cosh \frac{\Omega_{n i} x}{L_{X}}\right]\left[\sin \frac{\Omega_{n j} y}{L_{Y}}+A_{n j} \cos \frac{\Omega_{n j} y}{L_{Y}}+B_{n j} \sinh \frac{\Omega_{n j} y}{L_{Y}}+C_{n j} \cosh \frac{\Omega_{n j} y}{L_{Y}}\right]
\end{aligned}
$$

Equation (63) is the transverse displacement response to a moving mass of a rectangular plate resting on variable Winkler elastic foundation. The constants $A_{n i}, A_{p i}, A_{n j}, A_{p j}, B_{n i}, B_{p i}, B_{n j}, B_{p j}$, $\mathrm{C}_{\mathrm{ni}}, \mathrm{C}_{\mathrm{pi}}, \mathrm{C}_{\mathrm{nj}}$ and $\mathrm{C}_{\mathrm{pj}}$ are to be determined from the choice of the end support condition. 


\section{ANALYSIS OF THE SOLUTION}

Next, the phenomenon of resonance is examined. Equation (57) clearly shows that the rectangular plate on a variable Winkler elastic foundation and traversed by a moving force reaches a state of resonance whenever

$$
\gamma_{s f}=\frac{\Omega_{p i} c}{L_{X}}
$$

while equation (63) shows that the same plate under the action of a moving mass experiences resonance effect whenever

$$
\beta_{s f}=\frac{\Omega_{p i} c}{L_{X}}
$$

where

$$
\beta_{s f}=\gamma_{s f}\left[1-\frac{\mu_{0}}{2}\left(R_{1}-\frac{R_{3}}{\gamma_{s f}^{2}}\right)\right]
$$

Equations (65) and (66) imply that

$$
\beta_{s f}=\gamma_{s f}\left[1-\frac{\mu_{0}}{2}\left(R_{1}-\frac{R_{3}}{\gamma_{s f}^{2}}\right)\right]=\frac{\Omega_{p i} c}{L_{X}}
$$

Consequently from equations (64) and (67), for the same natural frequency, the critical speed (and the natural frequency) for the system traversed by a moving mass is smaller than that of the same system traversed by a moving force. Thus, for the same natural frequency of the plate, the resonance is reached earlier when we consider the moving mass system than when we consider the moving force system.

\section{ILLUSTRATIVE EXAMPLES}

a. Rectangular plate elastically supported at edges $y=0, y=L_{Y}$ with simple support at edges $x=0, x=L_{x}$.

At $\mathrm{x}=0$ and $\mathrm{x}=\mathrm{L}_{\mathrm{X}}$, the plate is taken to be simply supported and at the edges $\mathrm{y}=0$ and $\mathrm{y}=$ $\mathrm{L}_{\mathrm{Y}}$, it is taken to be elastically supported.

Using the conditions (4-11) in equations (34) and (35), the following values of the constants and the frequency equation are obtained for the elastic edges. 


$$
\begin{gathered}
C_{n j}=\frac{\left[\frac{\Omega_{n j}}{L_{Y}}-k_{1} r_{2}\right] \sin \Omega_{n j}+\left[k_{1}+\frac{r_{2} \Omega_{n j}}{L_{Y}}\right] \cos \Omega_{n j}-\frac{r_{1} \Omega_{n j}}{L_{Y}} \sinh \Omega_{n j}+k_{1} r_{1} \cosh \Omega_{n j}}{k_{1} r_{1} \sin \Omega_{n j}-\frac{r_{1} \Omega_{n j}}{L_{Y}} \cos \Omega_{n j}+\left[\frac{r_{3} \Omega_{n j}}{L_{Y}}-k_{1}\right] \sinh \Omega_{n j}+\left[\frac{\Omega_{n j}}{L_{Y}}-k_{1} r_{3}\right] \cosh \Omega_{n j}} \\
=\frac{-\left[\frac{r_{2} \Omega_{n j}^{3}}{L_{Y}^{3}}+k_{2}\right] \sin \Omega_{n j}+\left[\frac{\Omega_{n j}^{3}}{L_{Y}^{3}}-k_{2} r_{2}\right] \cos \Omega_{n j}-k_{2} r_{1} \sinh \Omega_{n j}-\frac{r_{1} \Omega_{n j}^{3}}{L_{Y}^{3}} \cosh \Omega_{n j}}{\frac{r_{1} \Omega_{n j}^{3}}{L_{Y}^{3}} \sin \Omega_{n j}+k_{2} r_{1} \cos \Omega_{n j}+\left[\frac{\Omega_{n j}^{3}}{L_{Y}^{3}}+k_{2} r_{3}\right] \sinh \Omega_{n j}+\left[\frac{r_{3} \Omega_{n j}^{3}}{L_{Y}^{3}}+k_{2}\right] \cosh \Omega_{n j}}, \\
A_{n j}=r_{1} C_{n j}+r_{2} \text { and } B_{n j}=r_{3} C_{n j}+r_{1}
\end{gathered}
$$

where

$$
r_{1}=\frac{\frac{\Omega_{n j}^{4}}{L_{Y}^{4}}+k_{1} k_{2}}{\frac{\Omega_{n j}^{4}}{L_{Y}^{4}}-k_{1} k_{2}} ; \quad r_{2}=\frac{-\frac{2 k_{1} \Omega_{n j}^{3}}{L_{Y}^{3}}}{\frac{\Omega_{n j}^{4}}{L_{Y}^{4}}-k_{1} k_{2}} \text { and } r_{3}=\frac{\frac{-2 k_{2} \Omega_{n j}}{L_{Y}}}{\frac{\Omega_{n j}^{4}}{L_{Y}^{4}}-k_{1} k_{2}} \text {. }
$$

Equation (68) when simplified yields

$$
\tan \Omega_{n j}=\tanh \Omega_{n j}
$$

which is termed the frequency equation for the elastic edge, such that

$$
\Omega_{1}=3.927, \Omega_{2}=7.069, \Omega_{3}=10.210, \ldots
$$

For the simple edges, it can be shown that

$$
\mathrm{A}_{\mathrm{ni}}=0, \mathrm{~B}_{\mathrm{ni}}=0, \mathrm{C}_{\mathrm{ni}}=0 \text {, and } \Omega_{\mathrm{ni}}=\mathrm{n}_{\mathrm{i}} \pi
$$

Similarly,

$$
\mathrm{A}_{\mathrm{pi}}=0, \mathrm{~B}_{\mathrm{pi}}=0, \mathrm{C}_{\mathrm{pi}}=0, \text { and } \Omega_{\mathrm{pi}}=\mathrm{p}_{\mathrm{i}} \pi
$$

Using (68), (69), (71), (72) and (73) in equations (57) and (63) one obtains the displacement response respectively to a moving force and a moving mass of a simple-elastic rectangular plate resting on a variable Winkler elastic foundation. 


\section{b. Elastic support at all edges.}

Using the conditions (12-19) in equations (34) and (35), one obtains

$$
\begin{gathered}
C_{n i}=\frac{\left[\frac{\Omega_{n i}}{L_{X}}-k_{1} r_{2}(i)\right] \sin \Omega_{n i}+\left[k_{1}+\frac{r_{2}(i) \Omega_{n i}}{L_{X}}\right] \cos \Omega_{n i}-\frac{r_{1}(i) \Omega_{n i}}{L_{X}} \sinh \Omega_{n i}+k_{1} r_{1}(i) \cosh \Omega_{n i}}{k_{1} r_{1}(i) \sin \Omega_{n i}-\frac{r_{1}(i) \Omega_{n i}}{L_{X}} \cos \Omega_{n i}+\left[\frac{r_{3}(i) \Omega_{n i}}{L_{X}}-k_{1}\right] \sinh \Omega_{n i}+\left[\frac{\Omega_{n i}}{L_{X}}-k_{1} r_{3}(i)\right] \cosh \Omega_{n i}} \\
=\frac{-\left[\frac{r_{2}(i) \Omega_{n i}^{3}}{L_{X}^{3}}+k_{2}\right] \sin \Omega_{n i}+\left[\frac{\Omega_{n i}^{3}}{L_{X}^{3}}-k_{2} r_{2}(i)\right] \cos \Omega_{n i}-k_{2} r_{1}(i) \sinh \Omega_{n i}-\frac{r_{1}(i) \Omega_{n i}^{3}}{L_{X}^{3}} \cosh \Omega_{n i}}{\frac{r_{1}(i) \Omega_{n i}^{3}}{L_{X}^{3}} \sin \Omega_{n i}+k_{2} r_{1}(i) \cos \Omega_{n i}+\left[\frac{\Omega_{n i}^{3}}{L_{X}^{3}}+k_{2} r_{3}(i)\right] \sinh \Omega_{n i}+\left[\frac{r_{3}(i) \Omega_{n i}^{3}}{L_{X}^{3}}+k_{2}\right] \cosh \Omega_{n i}}, \\
A_{n i}=r_{1}(i) C_{n i}+r_{2}(i) \text { and } B_{n i}=r_{3}(i) C_{n i}+r_{1}(i)
\end{gathered}
$$

where

$$
r_{1}(i)=\frac{\frac{\Omega_{n i}^{4}}{L_{X}^{4}}+k_{1} k_{2}}{\frac{\Omega_{n i}^{4}}{L_{X}^{4}}-k_{1} k_{2}} ; \quad r_{2}(i)=\frac{-\frac{2 k_{1} \Omega_{n i}^{3}}{L_{X}^{3}}}{\frac{\Omega_{n i}^{4}}{L_{X}^{4}}-k_{1} k_{2}} \text { and } r_{3}(i)=\frac{\frac{-2 k_{2} \Omega_{n i}}{L_{X}}}{\frac{\Omega_{n i}^{4}}{L_{X}^{4}}-k_{1} k_{2}}
$$

and

$$
\begin{gathered}
C_{n j}=\frac{\left[\frac{\Omega_{n j}}{L_{Y}}-k_{1} r_{2}(j)\right] \sin \Omega_{n j}+\left[k_{1}+\frac{r_{2}(j) \Omega_{n j}}{L_{Y}}\right] \cos \Omega_{n j}-\frac{r_{1}(j) \Omega_{n j}}{L_{Y}} \sinh \Omega_{n j}+k_{1} r_{1}(j) \cosh \Omega_{n j}}{k_{1} r_{1}(j) \sin \Omega_{n j}-\frac{r_{1}(j) \Omega_{n j}}{L_{Y}} \cos \Omega_{n j}+\left[\frac{r_{3}(j) \Omega_{n j}}{L_{Y}}-k_{1}\right] \sinh \Omega_{n j}+\left[\frac{\Omega_{n j}}{L_{Y}}-k_{1} r_{3}(j)\right] \cosh \Omega_{n j}} \\
=\frac{-\left[\frac{r_{2}(j) \Omega_{n j}^{3}}{L_{Y}^{3}}+k_{2}\right] \sin \Omega_{n j}+\left[\frac{\Omega_{n j}^{3}}{L_{Y}^{3}}-k_{2} r_{2}(j)\right] \cos \Omega_{n j}-k_{2} r_{1}(j) \sinh \Omega_{n j}-\frac{r_{1}(j) \Omega_{n j}^{3}}{L_{Y}^{3}} \cosh \Omega_{n j}}{\frac{r_{1}(j) \Omega_{n j}^{3}}{L_{Y}^{3}} \sin \Omega_{n j}+k_{2} r_{1}(j) \cos \Omega_{n j}+\left[\frac{\Omega_{n j}^{3}}{L_{Y}^{3}}+k_{2} r_{3}(j)\right] \sinh \Omega_{n j}+\left[\frac{r_{3}(j) \Omega_{n j}^{3}}{L_{Y}^{3}}+k_{2}\right] \cosh \Omega_{n j}}, \\
A_{n j}=r_{1}(j) C_{n j}+r_{2}(j) \text { and } B_{n j}=r_{3}(j) C_{n j}+r_{1}(j)
\end{gathered}
$$

where 


$$
r_{1}(j)=\frac{\frac{\Omega_{n j}^{4}}{L_{Y}^{4}}+k_{1} k_{2}}{\frac{\Omega_{n j}^{4}}{L_{Y}^{4}}-k_{1} k_{2}} ; \quad r_{2}(j)=\frac{-\frac{2 k_{1} \Omega_{n j}^{3}}{L_{Y}^{3}}}{\frac{\Omega_{n j}^{4}}{L_{Y}^{4}}-k_{1} k_{2}} \text { and } r_{3}(j)=\frac{\frac{-2 k_{2} \Omega_{n j}}{L_{Y}}}{\frac{\Omega_{n j}^{4}}{L_{Y}^{4}}-k_{1} k_{2}} \text {. }
$$

Equations (74) and (76) when simplified yield

$$
\tan \Omega_{n i}=\tanh \Omega_{n i}
$$

and

$$
\tan \Omega_{n j}=\tanh \Omega_{n j}
$$

Using (74), (75), (76), (77), (78) and (79) in equations (57) and (63) one obtains the transverse-displacement response respectively to a moving force and a moving mass of an elastically supported rectangular plate resting on a variable Winkler elastic foundation.

\section{NUMERICAL CALCULATIONS AND DISCUSSION OF RESULTS}

In order to carry out the calculations of practical interests in dynamics of structures and engineering design for the elastically supported plate resting on variable Winkler elastic foundation, a rectangular plate of length $\mathrm{L}_{\mathrm{Y}}=0.914 \mathrm{~m}$ and breadth $\mathrm{L}_{\mathrm{X}}=0.457 \mathrm{~m}$ is considered. It is assumed that the mass travels at the constant velocity $0.8123 \mathrm{~m} / \mathrm{s}$. Furthermore, values for $\mathrm{E}, \mathrm{S}$ and $\Gamma$ are chosen to be $2.109 \times 10^{9} \mathrm{~kg} / \mathrm{m}^{2}, 0.4 \mathrm{~m}$ and 0.2 respectively. For various values of the foundation modulus $\mathrm{F}_{0}$ and the rotatory inertia correction factor $\mathrm{R}_{0}$, the deflections $\mathrm{W}(\mathrm{x}, \mathrm{y}, \mathrm{t})$ of the elastically supported plate are calculated in meters at $x=\frac{L_{X}}{2}$ and $y=\frac{L_{Y}}{2}$ and plotted against time t in seconds.

\section{a. Simple - elastic rectangular plate on variable Winkler foundation.}

Figures $6.1-6.3$ present the responses of the plate simply supported at the edges $\mathrm{x}=0$ and $\mathrm{x}=$ $\mathrm{L}_{\mathrm{X}}$ and elastically supported at the edges $\mathrm{y}=0$ and $\mathrm{y}=\mathrm{L}_{\mathrm{Y}}$. Figure 6.1 displays the effect of foundation modulus $\mathrm{F}_{0}$ on the transverse deflection of moving force for simple-elastic rectangular plate, while figure 6.2 displays the effect of rotatory inertia correction factor $\mathrm{R}_{0}$ on the transverse displacement of moving mass for the simple-elastic plate at a fixed value of $F_{0}=1000 \mathrm{~N} / \mathrm{m}^{3}$. It is shown that as both $\mathrm{F}_{0}$ and $\mathrm{R}_{0}$ increase the amplitude of the deflection decreases respectively for the simple-elastic rectangular plate resting on variable Winkler elastic foundation. 


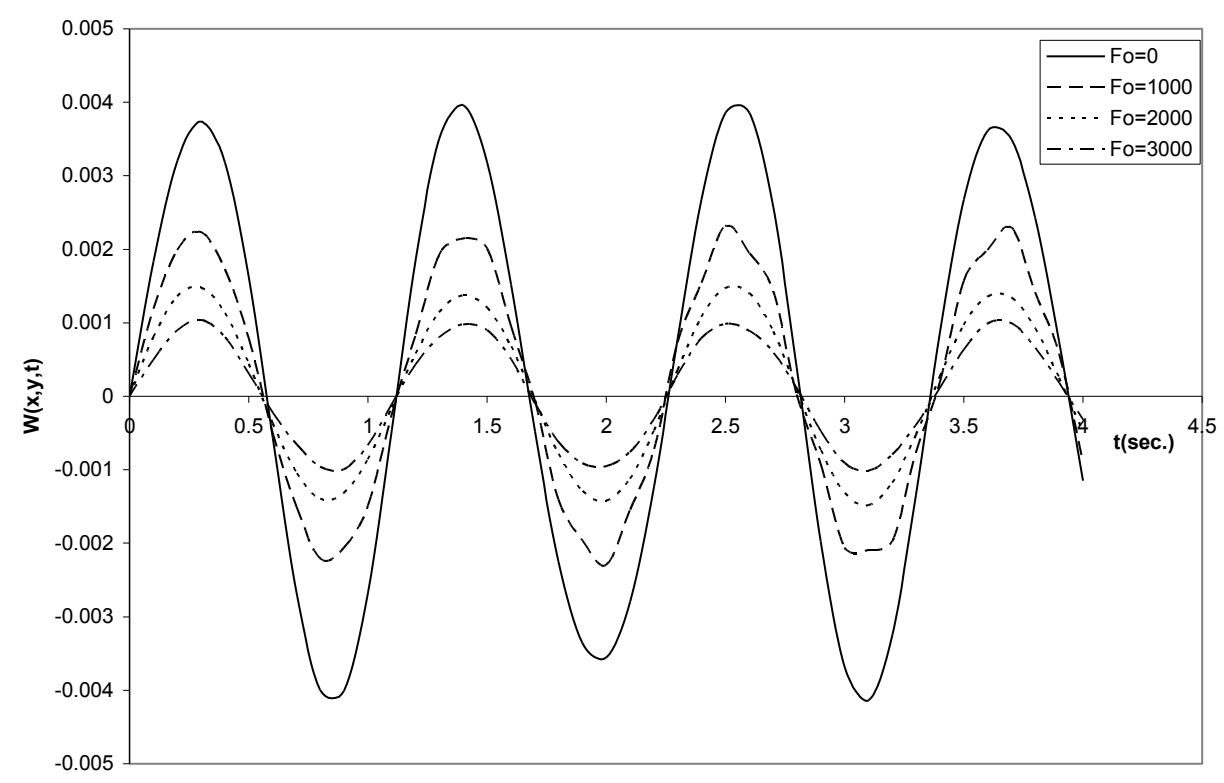

Figure 6.1 Displacement of moving force for simple-elalastic rectangular plate on variable Winkler foundation for various values of foundation modulus Fo.

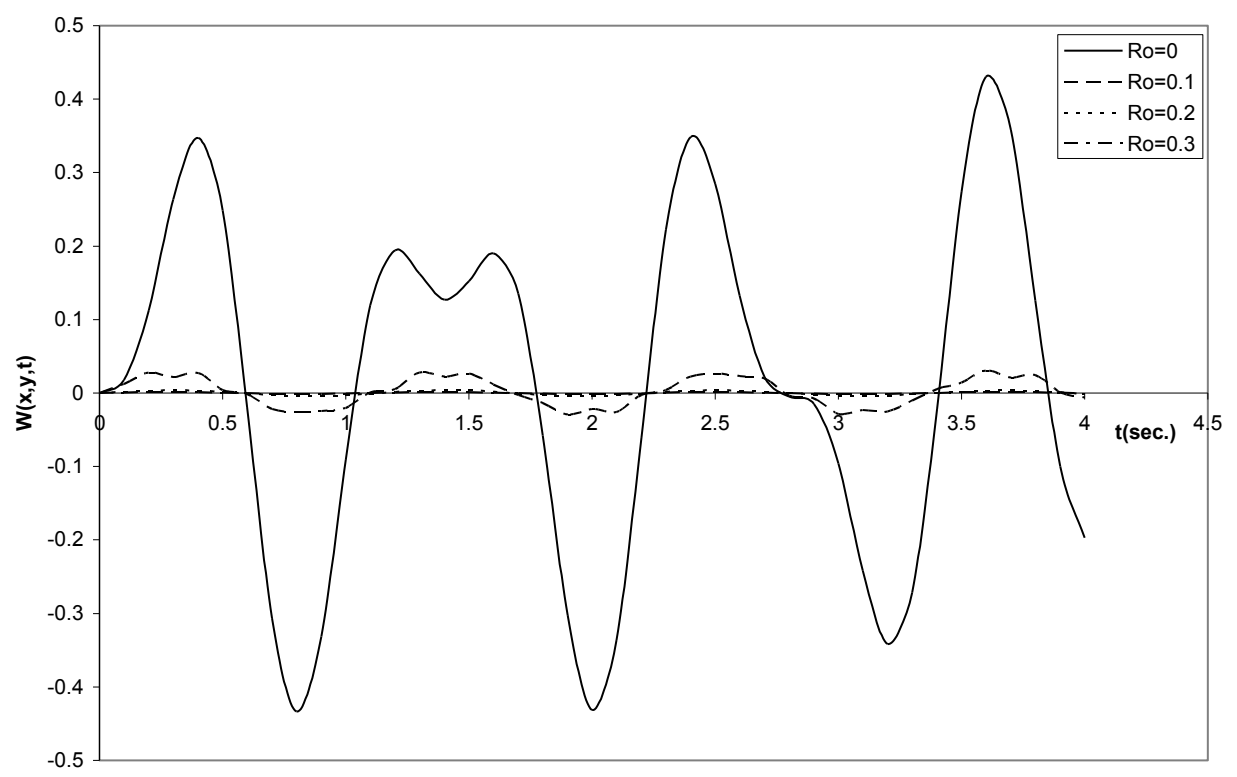

Figure 6.2 Displacement of moving mass for simple-elalastic rectangular plate on variable Winkler foundation for various values of rotatory inertia correction factor Ro. 
For the purpose of comparison, Figure 6.3 compares the displacement curves of moving force and moving mass for the simple - elastic plate for fixed $F_{0}$ and $R_{0}$. It is evident from the graph that the response amplitude of a moving mass is greater than that of a moving force problem.

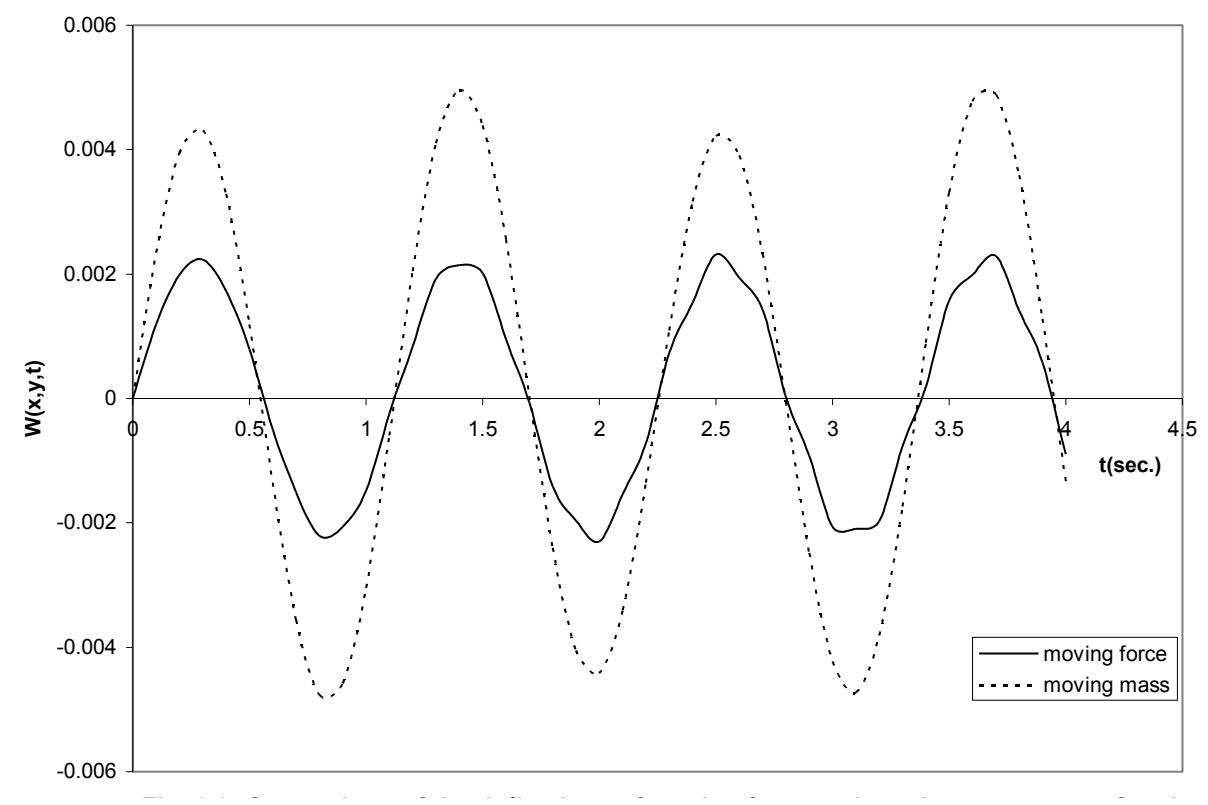

Figure 6.3 Comparison of the deflections of moving force and moving mass cases for simple-elastic rectangular plate on variable Winkler foundation with $\mathrm{Fo}_{\mathrm{o}}=1000$ and $\mathrm{Ro}=4$.

\section{b. Elastically supported rectangular plate on variable Winkler foundation.}

The responses of the plate elastically supported at all its edges are presented in figures $6.4-6.6$. observed in figures 6.4 and 6.5 that as the values of $\mathrm{R}_{0}$ and $\mathrm{F}_{0}$ increase the deflection amplitude of the plate decreases for both cases of moving force and moving mass respectively for fixed $\mathrm{F}_{0}=$ $1000 \mathrm{~N} / \mathrm{m}^{3}$ in figure 6.4. Figure 6.6 compares the displacement response of the moving force and moving mass for an elastically supported rectangular plate for fixed values of $F_{0}$ and $R_{0}$. It is evident that the displacement response of the moving mass problem is greater than that of the moving force problem. 


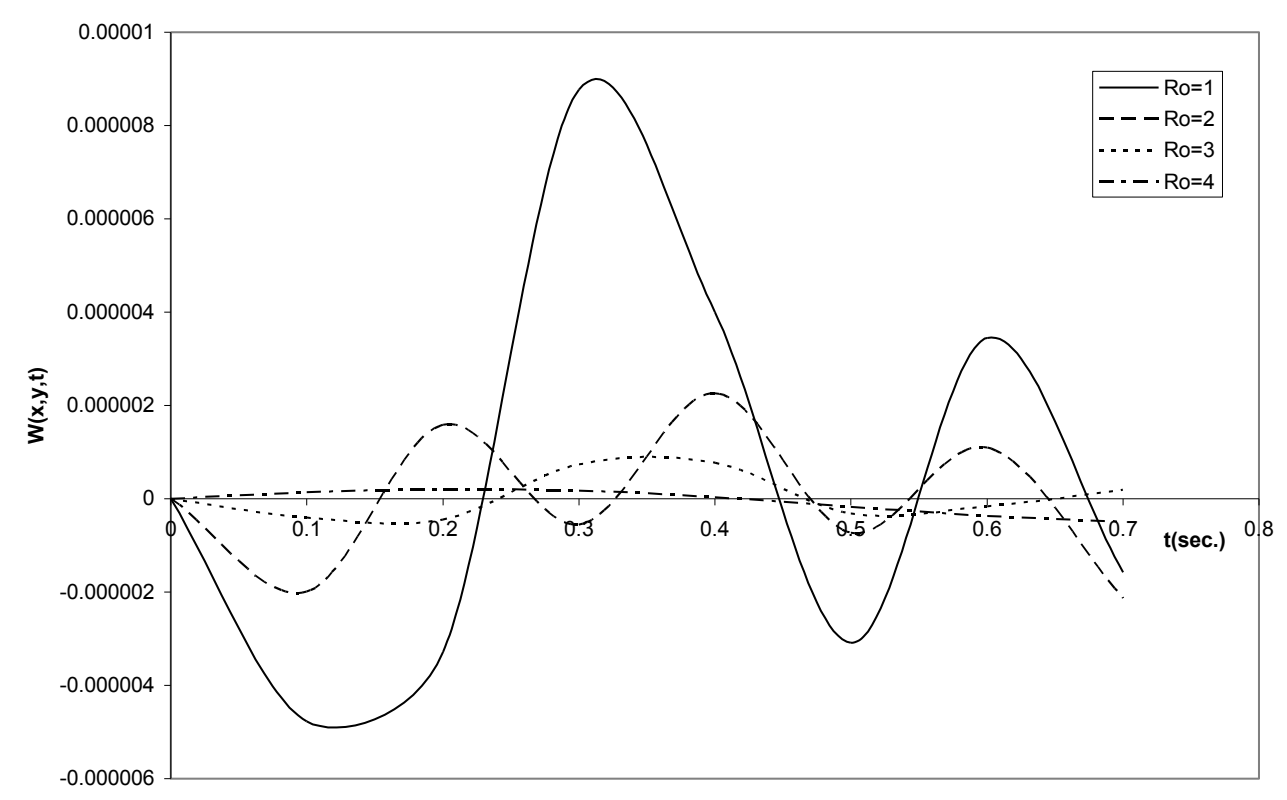

Figure 6.4 Deflection profile of moving force for elastically supported rectangular plate on variable Winkler foundation for various values of rotatory inertia correction factor Ro.

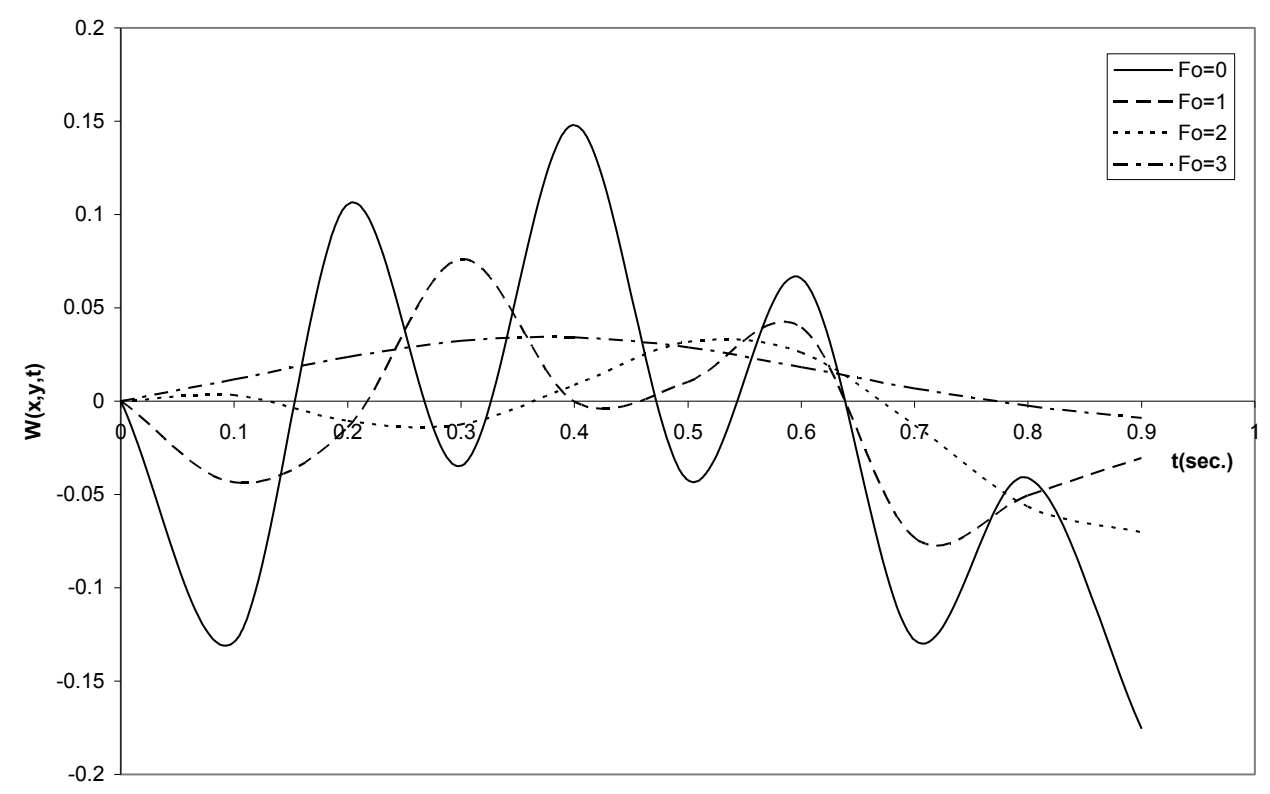

Figure 6.5 Deflection profile of moving mass for elastically supported rectangular plate on variable Winkler for various values of foundation modulus Fo. 


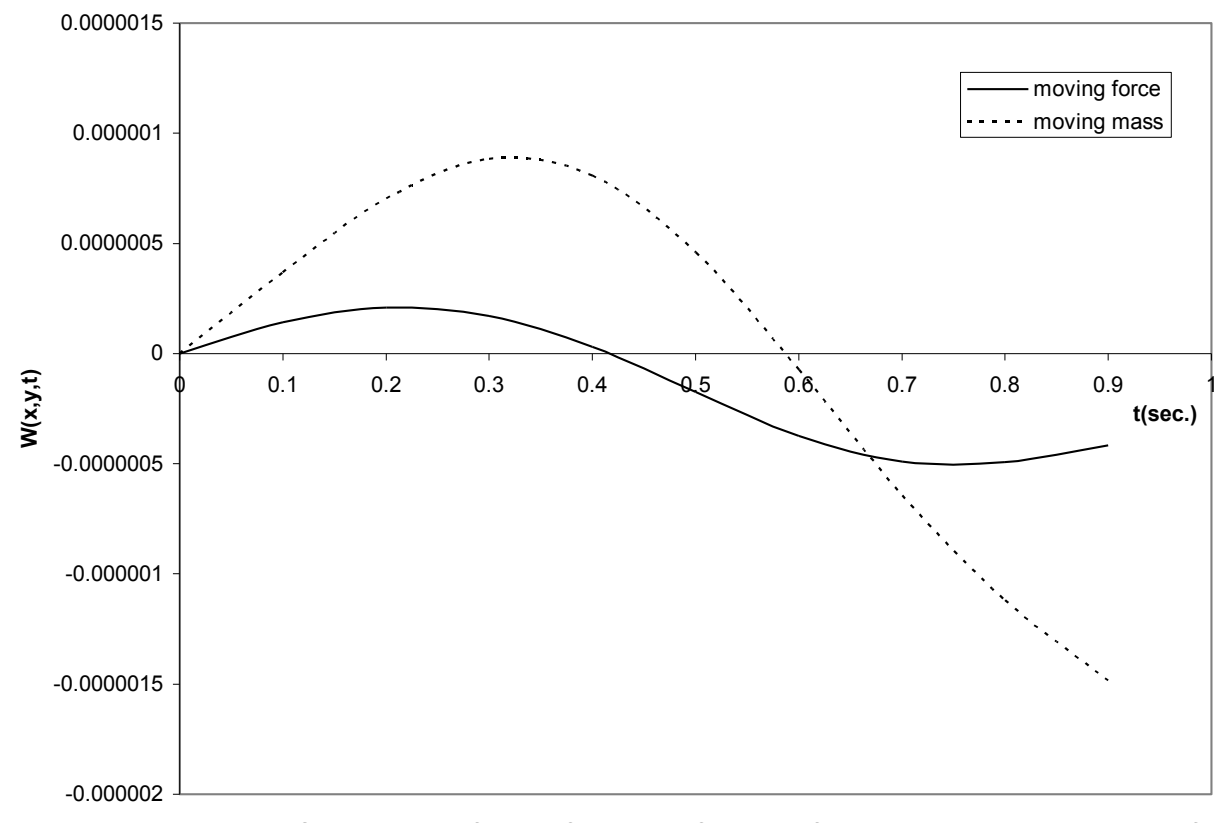

Figure 6.6 Comparison of the deflections of moving force and moving mass cases for elastically supported plate on variable Winkler foundation with $\mathrm{F}_{\mathrm{o}}=1000$ and $\mathrm{Ro}=4$.

\section{CONCLUSION}

The problem of the dynamic behaviour under moving concentrated masses of rectangular plates resting on variable Winkler elastic foundation is considered in this work. The governing fourth order partial differential equation is a non-homogenous equation with variable and singular coefficients. The objective of the work has been to study the problem of the dynamic response to moving concentrated masses of rectangular plates on variable Winkler elastic foundations. In particular, the closed form solutions of the fourth order partial differential equations with variable and singular coefficients of the rectangular plate is obtained for both cases of moving force and moving mass. The method is based on (i) Separation of variables (ii) The modified Struble's technique and (iii) The method of integral transformations.

These solutions are analyzed and resonance conditions are obtained for the problem. The numerical analysis for both moving force and moving mass problems carried out show that the moving force solution is not an upper bound for the accurate solution of the moving mass problem and that as the rotatory inertia correction factor increases, the response amplitudes of the plates decrease for both cases of moving force and moving mass problem. When the rotatory inertia correction factor is fixed, the displacements of the elastically supported rectangular plates resting on variable Winkler elastic foundations decrease as the foundation modulus increases.

Furthermore, for fixed values of rotatory inertia correction factor and foundation modulus, the response amplitude for the moving mass problem is greater than that of the moving force problem implying that resonance is reached earlier in moving mass problem than in moving force problem of the elastically supported rectangular plate resting on variable Winkler foundation. Hence, it is dangerous to rely on the moving force solutions.

Latin American Journal of Solids and Structures 11 (2014) 1515-1540 
Finally, for the elastically supported rectangular plate resting on Winkler elastic foundation with stiffness variation, for the same natural frequency, the critical speed for moving mass problem is smaller than that of the moving force problem, and as rotatory inertia correction factor and the foundation modulus increase, the critical speeds increase showing that risk is reduced.

\section{References}

Adams, G. G. (1995): Critical speeds and the response of a tensioned beam on an elastic foundation to repetitive moving loads. Int. Jour. Mech. Sci., Vol. 7, pp $773-781$.

Clastornic, J. Eisenberger, M. Yankelevsky, D. Z. and Adin, M. A. (1986): Beams on variable elastic foundation. Journal of Applied Mechanics. Vol. 53. pp 925-928.

Douglas Gregory, R. Charles C. Gu and Frederic Y. M. Wan. (2002): The cantilever strip plate of varying thickness and the centre of shear. Q. JI Mech. Appl. Math. Vol. 55(1). pp 29-48.

Franklin, J. N. and Scott, R. F. (1979): Beam equation with variable foundation coefficient. ASCE. Eng. Mech. Div. Vol. 105, EMS, pp 811-827.

Gbadeyan, J. A. and Oni, S. T. (1995): Dynamic behaviour of beams and rectangular plates under moving loads. Journal of Sound and Vibration 182(5), pp 677-693.

Jia-Jang Wu (2006): Vibration analysis of a portal frame under the action of a moving distributed mass using moving mass element. Int. Jour. for Numerical Methods in Engineering, 62, pp 2028 - 2052.

Lee, H. P. and Ng, T. Y. (1996): Transverse vibration of a plate moving over multiple point supports. Applied Acoustics, Vol. 47, No. 4, pp $291-301$.

Muscolino, G. and Palmeri, A. (2007): Response of beams resting on viscoelastically damped foundation to moving oscillators. International Journal of Solids and Structures. 44(5). pp 1317-1336.

Milornir, M., Stanisic, M. and Hardin, J. C. (1969): On the response of beams to an arbitrary number of concentrated moving masses. Journal of the franklin institute, Vol. 287, No 2.

Omer Civalek and Aitung Yauas (2006): Large deflection static analysis of rectangular plates on two parameter elastic foundations. International Journal of Science and Technology. Vol. 1, No. 1, pp $43-50$.

Oni, S. T. and Awodola, T. O. (2003): Vibrations under a moving load of a non-uniform Rayleigh beam on variable elastic foundation. Journal of Nigerian Association of Mathematical Physics, Vol. 7, pp $191-206$.

Oni, S. T. (2004): Flexural motions of a uniform beam under the actions of a concentrated mass traveling with variable velocity. Abacus, Journal of Mathematical Association of Nigeria. Vol. 31, No 2a, pp 79 - 93.

Oni, S. T. and Awodola, T. O. (2005): Dynamic response to moving concentrated masses of uniform Rayleigh beams resting on variable Winkler elastic foundation. Journal of the Nigerian Association of Mathematical Physics. Vol. 9. pp 151-162.

Oni, S. T. and Omolofe, B. (2005): Dynamic analysis of a prestressed elastic beam with general boundary conditions under moving loads at varying velocities. Journal of Engineering and Engineering Technology,FUTA,Vol. 4,No1, pp55-72.

Oni, S. T. and Awodola, T. O. (2010): Dynamic response under a moving load of an elastically supported non-prismatic Bernoulli-Euler beam on variable elastic foundation. Latin American Journal of Solids and Structures. 7, pp $3-20$.

Oni, S. T. and Awodola, T. O. (2011): Dynamic behaviour under moving concentrated masses of simply supported rectangular plates resting on variable Winkler elastic foundation. Latin American Journal of Solids and Structures (LAJSS), vol. 8, pp 373-392. 
Pesterev, A. V. Tan, C. A. Bergman, L. A. (2001): A new method for calculating bending moment and shear force in moving load problems. Journal of Applied mechanics. Vol. 68. pp 252-259.

Sadiku, S. and Leipholz, H. H. E. (1981): On the dynamics of elastic systems with moving concentrated masses. Ing. Archiv. 57, pp 223-242.

Savin, E. (2001): Dynamics amplification factor and response spectrum for the evaluation of vibrations of beams under successive moving loads. Journal of Sound and Vibrations, 248(2), pp 267 - 288.

Shadnam, M. R., Mofid, M. and Akin, J. E. (2001): On the dynamic response of rectangular plate, with moving mass. Thin-Walled Structures, 39(2001), pp $797-806$.

Stanisic, M.M., Euler, J.A. and Montgomery, S.T. (1974): On a theory concerning the dynamical behaviour of structures carrying moving masses. Ing. Archiv. 43, pp. 295-303.

Wilson, J. F. (1974): Dynamic whip of elastically restrained plate strip to rapid transit loads. Transactions of the American Society of Mechanical Engineers. Series G. 96. pp 163-168. 